\title{
Estimation of Visceral Fat and Fatty Liver Disease Using Ultrasound in Patients with Diabetes
}

\author{
Yasunori Onitsuka ${ }^{1}$, Fuminao Takeshima ${ }^{2}$, Tatsuki Ichikawa ${ }^{2}$, \\ Shigeru Kohno ${ }^{3}$ and Kazuhiko Nakao ${ }^{2}$
}

\begin{abstract}
Objective Fatty liver disease is the most commonly encountered form of chronic liver dysfunction in routine medical care and is closely associated with type 2 diabetes. We aimed to elucidate how the use of new medications affects the incidence of fatty liver disease and amount of visceral fat, both of which are associated with diabetes.

Methods Abdominal ultrasonography was performed to assess the preperitoneal fat thickness (PFT) and presence of fatty liver. The PFT, body mass index (BMI) and waist circumference were used to investigate the rate and development of fatty liver disease in each group. A multivariate analysis with multiple logistic regression was performed using the PFT and presence of fatty liver disease as dependent variables.

Patients We evaluated 202 patients treated at the Onitsuka Clinic. The subjects were divided into three subgroups (non-diabetic and diabetic with or without treatment with antidiabetic medications).

Results Positive correlations between the PFT, BMI, and waist circumference were observed. No increases in the prevalence of fatty liver disease were observed in the medicated diabetic group, even when the PFT levels were high. A multivariate analysis with multiple logistic regression revealed that visceral fat accumulation was inhibited in women and those taking statins or thiazolidines and aggravated in men and those with obesity or an increased waist circumference. Obesity was an aggravating factor for fatty liver disease, and biguanides were useful as counteractants.

Conclusion Measuring the PFT is effective for screening metabolic syndrome and evaluating diabetes, dyslipidemia and hypertension associated with fatty liver disease. Clinically, fatty liver progression to nonalcoholic steatohepatitis (NASH) may be prevented by tackling obesity and administering appropriate medications.
\end{abstract}

Key words: fatty liver disease, ultrasonography, preperitoneal fat thickness

(Intern Med 53: 545-553, 2014)

(DOI: 10.2169/internalmedicine.53.1294)

\section{Introduction}

Fatty liver disease is the most commonly encountered form of chronic liver dysfunction in routine medical care and is closely associated with type 2 diabetes. Its prevalence is increasing in Japan as more people adopt a Western lifestyle (1). The "two hits theory," proposed in 1998, argues that non-alcoholic steatohepatitis (NASH) arises in two stages. The "first hit" involves the increased hepatocellular uptake of free fatty acids, enhanced fatty acid synthesis associated with an increased flow of carbohydrates to liver cells and the impaired synthesis or decreased release of VLDL (very-low-density lipoproteins) in the liver, leading to simple fatty liver disease. The "second hit" follows at a later point and includes the following key processes that trigger the onset of NASH: oxidative stress, free radical generation, lipid peroxidation, inflammatory cytokine induction by en-

\footnotetext{
${ }^{1}$ Onitsuka Internal Medicine and Gastroenterology Clinic, Japan, ${ }^{2}$ Department of Gastroenterology and Hepatology, Graduate School of Biomedical Sciences, Nagasaki University, Japan and ${ }^{3}$ The Second Department of Internal Medicine, Graduate School of Biomedical Sciences, Nagasaki University, Japan

Received for publication July 4, 2013; Accepted for publication September 26, 2013

Correspondence to Dr. Fuminao Takeshima, ftake@ nagasaki-u.ac.jp
} 
Table 1. Characteristics of Subjects $(n=202)$

A)

\begin{tabular}{|c|c|c|c|c|c|c|c|c|c|c|c|}
\hline & \multicolumn{2}{|c|}{$\begin{array}{c}\text { N.S } \\
\text { ( Chi-square test) }\end{array}$} & \multicolumn{2}{|c|}{$\begin{array}{l}\text { Age, } \mathrm{p}=0.9641 . \mathrm{Ht}, \mathrm{p}=\mathrm{C} \\
\text { (Kruskal-Wallis test) }\end{array}$} & \multirow[b]{2}{*}{$\mathrm{Wt}(\mathrm{Kg})$} & BMI, $\mathrm{p}=0.0118 . \mathrm{W}$ & .C, $\mathrm{p}=0.0131$ & IbAlc, $\mathrm{p}<0.000$ & \multicolumn{3}{|c|}{ LDL, $\mathrm{p}<0.0001 . \mathrm{HDL}, \mathrm{p}=0.4456 . \mathrm{TG}, \mathrm{p}=0.1046$} \\
\hline & male & female & Age & $\mathrm{Ht}(\mathrm{cm})$ & & $\operatorname{BMI}(\mathrm{kg} / \mathrm{m} / \mathrm{m})$ & W.C $(\mathrm{cm})$ & $\mathrm{HbAlc}(\%)$ & $\mathrm{LDL}(\mathrm{mg} / \mathrm{dL})$ & $\mathrm{HDL}(\mathrm{mg} / \mathrm{dL})$ & $\mathrm{TG}(\mathrm{mg} / \mathrm{dL})$ \\
\hline non-DM & 37 & 65 & 72.2 & 153.9 & 58.7 & 24.57 & 85 & 25.57 & 95 & 56.3 & 109.8 \\
\hline non-medicated DM & 9 & 17 & 72.3 & 154.3 & 62.5 & 26.3 & 89.7 & $6.3^{\top}$ & 102.7 & 53.4 & 140.1 \\
\hline medicated DM & 33 & 41 & 72.5 & 153.6 & 61.3 & 26.1 & 89.2 & $6.5]$ & 80.3 」 & 56.1 & 125 \\
\hline
\end{tabular}

B)

BMI, $\mathrm{p}=0.0038$. W.C, $\mathrm{p}=0.0058$. HbAlc, $\mathrm{p}<0.0001$. LDL, $\mathrm{p}<0.0001$. ( 1 way-ANOVA, Tuky-Kramer test )

\begin{tabular}{crrrrc}
\hline $\mathrm{n}$ & \multicolumn{1}{c}{ ARB } & \multicolumn{1}{c}{ Ca-AT } & Esemitibe & Statin & Fibrate \\
\hline \hline non-DM & $56(54.9 \%)$ & $45(44.1 \%)$ & $8(7.8 \%)$ & $40(39.2 \%)$ & $5(4.9 \%)$ \\
non-medicated DM & $16(61.5 \%)$ & $15(57.7 \%)$ & $2(7.7 \%)$ & $12(46.2 \%)$ & $1(3.8 \%)$ \\
medicated DM & $52(70.3 \%)$ & $36(48.6 \%)$ & $4(5.4 \%)$ & $46(62.2 \%)$ & $1(1.4 \%)$ \\
(Chi-square test)
\end{tabular}

C)

\begin{tabular}{cccccccc}
\hline medication & DPP-4 & TZD & BG & a-GI & Gurind & SU & insulin \\
\hline \hline medicated DM & 58 & 21 & 22 & 5 & 1 & 15 & 7 \\
\hline
\end{tabular}

HT: Height, WT: Weight, BMI: Body mass index, W.C: Waist circumference, ARB: angiotensin II receptor blocker, Ca-AT: calcium antagonist, DPP-4: dipeptidyl-peptidase IV inhibitor, TZD: thiazolidinedione, BG: biguanide, a-GI: alpha-glucosidase inhibitor, SU: sulfonylurea

dotoxins, mitochondrial dysfunction and abnormal adipocytokine secretion (2).

Non-alcoholic fatty liver disease (NAFLD) refers to both simple fatty liver disease and NASH. Although the ratio of these diseases is generally considered to be 9:1, several reports have indicated that the proportion of NASH may actually be much higher (3). While NASH is relatively rare, liver cancer can arise from the resultant cirrhosis, and performing early intervention at the simple fatty liver disease stage, before progression to the second hit stage, is desirable.

Recently, NASH treatment has included Dipeptidyl Peptidase-4 (DPP-4) inhibitors in the list of effective medications (4-6). However, there are currently no studies evaluating therapeutic options for treating simple fatty liver disease and NASH in the general clinic setting. Therefore, we studied how these new medications affect the incidence of fatty liver disease and amount of visceral fat, which are associated with diabetes and progression to the second hit stage.

\section{Materials and Methods}

\section{Subjects}

All human investigations were performed after obtaining ethical approval by the Human Investigations Committee of the participating institutions. In this study, we examined a total of 202 patients, of whom 79 were men and 123 were women. The patients were divided into three groups. The first group consisted of 102 non-diabetics (non-DM group) who were primarily treated with essential medications for disorders such as hypertension, dyslipidemia and gastroesophageal reflux disease (GERD). The second group comprised 26 patients with mild diabetes treated using diet and exercise without antidiabetic medications who were concur- rently being medically treated for either hypertension or dyslipidemia (non-medicated DM group). The third group comprised 74 diabetic patients taking antidiabetic medications (medicated DM group).

There were no differences in sex, age, height, weight or the HDL-C (high-density lipoprotein cholesterol) or triglyceride levels between the groups. The BMI (body mass index) and HbA1c (glycosylated hemoglobin) values were significantly higher in the DM group $(\mathrm{p}<0.01)$, while the waist circumference values were significantly higher in the medicated DM group. Interestingly, the LDL-C (low-density lipoprotein cholesterol) levels were the lowest in the medicated DM group (Table 1A).

Statin treatment, consisting of either rosuvastatin, atorvastatin or pitavastatin, was more frequently administered in the DM groups than in the non-DM group; however, none of these medications significantly improved NASH. Furthermore, there were no differences between the groups treated with other medications previously reported to improve NASH, such as angiotensin receptor blockers, ezetimibe and bezafibrates. There were also no differences associated with the use of calcium antagonists for complications, such as hypertension and angina (Table 1B). Table 1C shows the medications taken by the patients in the medicated DM group.

\section{Methods}

The cutoff points for waist circumference were in accordance with the guidelines used for metabolic syndrome screening: $\geq 85 \mathrm{~cm}$ for men and $\geq 90 \mathrm{~cm}$ for women $(7,8)$. A Prosound $\alpha 10$ (ALOKA, Tokyo, Japan) ultrasound machine with a convex transducer was used for the ultrasound examinations. All ultrasound examinations and diagnoses were performed by the same internal medicine and gastroenterology specialist. Fatty liver disease was diagnosed in the presence of a hyperechoic liver and hepatorenal echo contrast on 


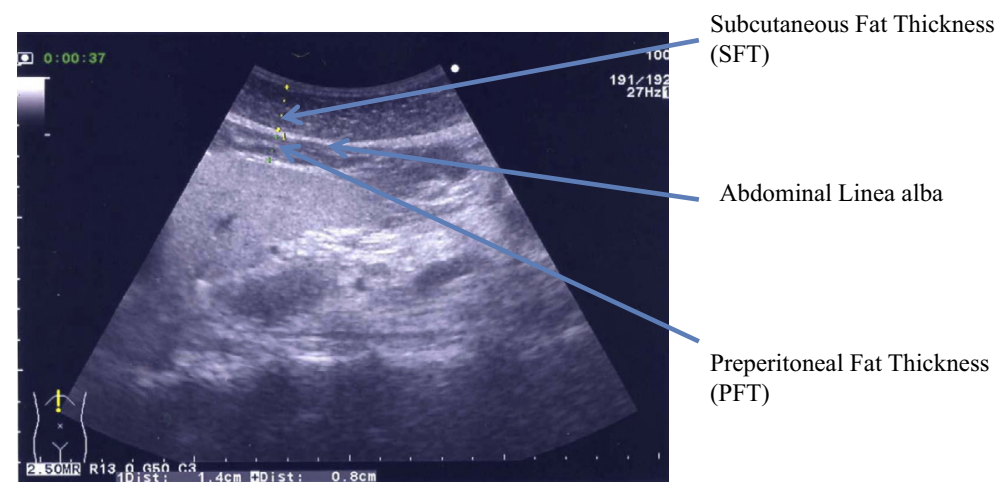

SFT1.4 PFT 0.8 Diagnosis by US: Fatty liver

Figure 1. Measurement of the PFT and SFT using abdominal ultrasonography in a fatty liver patient.

the ultrasound examination, regardless of whether the patient had abnormal liver function tests.

Ultrasound scanning was performed according to our standard protocol. Following the procedure described by Uchibori et al., a sagittal scan was obtained to visualize the left hepatic lobe, and the abdominal linea alba was scanned while keeping the surface of the liver as parallel to the skin as possible. The subcutaneous fat thickness (SFT) and preperitoneal fat thickness (PFT) were measured from the center of the left hepatic lobe (Fig. 1). The cutoff point for PFT was set at $\geq 0.8 \mathrm{~cm}$ based on the assumption that $\geq 0.8$ $\mathrm{cm}$ on an abdominal computed tomography (CT) scan corresponds to $100 \mathrm{~cm}^{2}$ of visceral fat (8-11).

\section{Statistical analysis}

The chi-square test was used to analyze differences in the sex ratio and medications between the different groups. The Kruskal-Wallis test and an analysis of variance (ANOVA) were used to analyze height, weight, BMI and the HbAlc, triglyceride (TG) and LDL levels. The Tukey-Kramer test was then used according to the multiple comparison method following a one way-ANOVA of BMI and the HbA1c and HDL levels. The PFT was analyzed using a three-way ANOVA before multiple comparison testing according to Ryan's method.

Due to the small number of cases (202), 90\% confidence intervals were used for the univariate analysis, multiple logistic regression analysis and multivariate analysis, while $95 \%$ confidence intervals were used for the other tests.

\section{Results}

\section{A positive correlation was found between the PFT, $B M I$ and waist circumference.}

Previous studies have indicated that the PFT is well correlated with the visceral fat volume and serum lipid levels. Our results also showed a strong positive correlation between the PFT and both BMI $(\mathrm{r}=0.534, \mathrm{p}<0.001)$ and waist circumference $(r=0.526, \mathrm{p}<0.001)$ (Fig. 2A).
In the DM group, there was a weak positive correlation between the PFT and BMI $(\mathrm{r}=0.449, \mathrm{p}<0.001)$ as well as a positive correlation between the PFT and waist circumference $(r=0.387, p<0.001)$ (Fig. 2B).

\section{No increases in the prevalence of fatty liver dis- ease were observed in the medicated DM group, even when the PFT was high.}

The prevalence of fatty liver disease in the non-DM $(73.3 \%)$ and non-medicated DM $(91.7 \%)$ groups was significantly increased in the patients with PFT $\geq 0.8(\mathrm{p}<0.0001)$; this trend was particularly marked in the latter group. In contrast, the prevalence of fatty liver disease remained the same in the medicated DM group (52.9\%), even when the PFT values were high. Furthermore, the prevalence of fatty liver disease was significantly lower in the medicated DM group than in the non-medicated DM group (Fig. 3).

\section{Statins and thiazolidinediones (TZD) reduced the PFT, while biguanides reduced the prevalence of fatty liver disease.}

The low prevalence of fatty liver disease observed in the medicated DM group suggests that some aspect of antidiabetic medication has an inhibitory effect on the prevalence of fatty liver disease, as well as an effect in improving fatty liver disease, in patients with obesity and visceral fat accumulation. The odds ratio for PFT and fatty liver disease was calculated using a univariate analysis.

Factors decreasing the risk of PFT (particularly the volume of mesenteric visceral fat) included a female sex (OR= $0.466)$ and taking antidiabetic medications $(\mathrm{OR}=0.769)$; the difference was significant for a female sex $(p=0.0198)$. In the diabetic patients, the aggravating factors were not taking antidiabetic medications $(\mathrm{OR}=2.115)$, mild obesity $(\mathrm{OR}=$ $4.984)$, moderate or severe obesity $(\mathrm{OR}=14.057)$ and a waist circumference over the established cutoff point $(\mathrm{OR}=5.714)$. Significant differences were found in all items $(p<0.0001)$, with the exception of treatment with/without medications (Fig. 4A). All medications for diabetes and hypertension had an inhibitory effect in decreasing the amount of visceral fat. 
A) Correlation between PFT and BMI or WC in the non-DM group
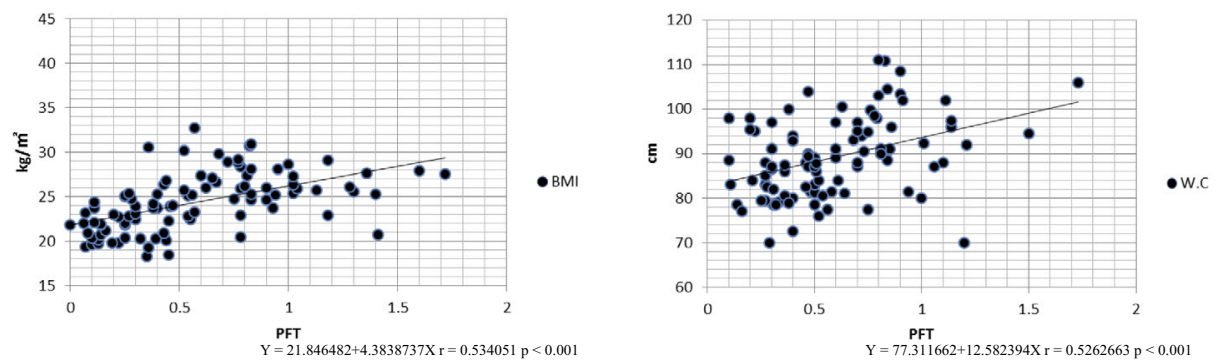

B) Correlation between PFT and BMI or WC in the DM group
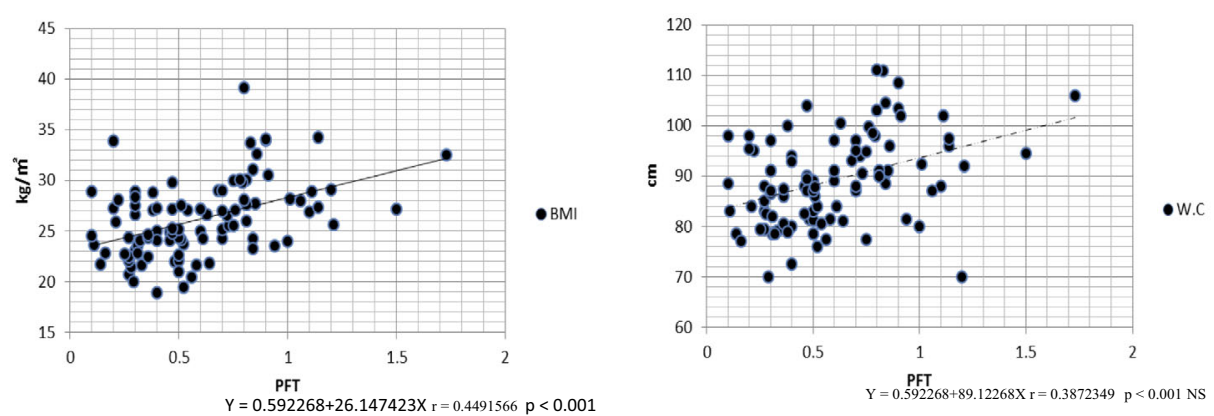

Figure 2. A: Correlations between the PFT and BMI or WC in the non-DM group. A significant correlation was observed between the PFT and BMI or $\mathrm{WC}$ in the non-DM group $(r=0.53, p<0.001$, $\mathbf{r}=0.53, \mathrm{p}<0.001$, respectively). B: Correlations between the PFT and BMI or WC in the DM group. A significant correlation was also observed between the PFT and BMI or WC in the DM group ( $r=0.45$, p $<0.001, r=0.39, p<0.001$, respectively). PFT: preperitoneal fat thickness BMI: body mass index WC: waist circumference

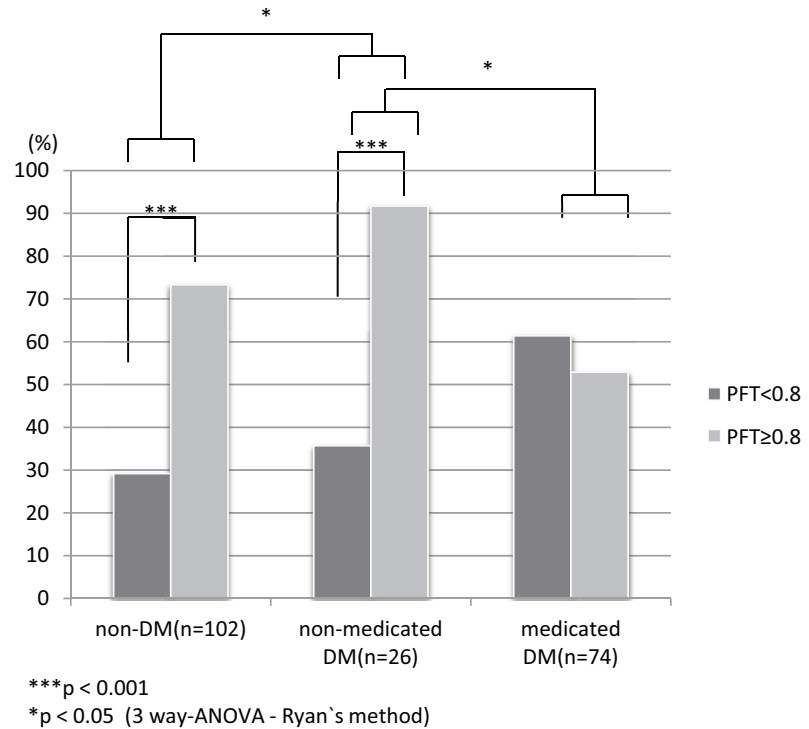

Figure 3. Prevalence of fatty liver disease in the non-DM group, non-medicated DM group and medicated DM group. Although the prevalence of fatty liver in both the non-DM and non-medicated DM groups was significantly increased among the patients with a high PFT $(\geq 0.8)$ compared with that observed in the patients with a low PFT $(<0.8)$, no increases were observed in the medicated DM group, even among the patients with a high PFT. PFT: preperitoneal fat thickness
Of the antihypertensive agents, calcium antagonists had no effect (OR=0.973), while angiotensin II receptor blocker (ARB) therapy increased the risk of developing visceral fat $(\mathrm{OR}=1.412)$. Significant differences were obtained with statin $(p=0.0236)$ and TZD ( $p=0.0686)$ therapy (Fig. 4B).

The risk of fatty liver disease was significantly increased by the following variables: the lack of antidiabetic medication $(\mathrm{OR}=2.192, \mathrm{p}=0.0866)$, mild obesity $(\mathrm{OR}=3.474, \mathrm{p}=$ $0.0001)$, moderate or severe obesity $(\mathrm{OR}=8.4, \mathrm{p}=0.0002)$ and waist circumference $(\mathrm{OR}=3.635, \mathrm{p}<0.0001)$. The sex ratio had no effect on the risk of developing fatty liver disease (Fig. 5A). Statins $(\mathrm{OR}=1.306)$ and $\mathrm{ARBs}(\mathrm{OR}=1.271)$ aggravated fatty liver disease, whereas DPP-4 $(\mathrm{OR}=0.739)$, insulin $(\mathrm{OR}=0.188)$, ezetimibe $(\mathrm{OR}=0.753)$, sulfonylureas $(\mathrm{SUs})$ $(\mathrm{OR}=0.477)$, TZD $(\mathrm{OR}=0.517)$ and biguanide $(\mathrm{BG}) \mathrm{s}(\mathrm{OR}=$ $0.395)$ each had an inhibitory effect. A significant difference was obtained for BGs (Fig. 5B). There was an insufficient number of patients taking alpha-glucosidase $(\alpha-\mathrm{GI})$ inhibitors or fibrates to make an analysis.

As there were many causal factors, a multivariate analysis with multiple logistic regression was performed using the PFT and presence of fatty liver disease as dependent variables. The multiple logistic regression analysis of PFT demonstrated that the following factors lead to increased visceral fat: diabetes, obesity, waist circumference over the cutoff point and ARB therapy. The following had an inhibitory effect on visceral fat accumulation (Table 2): a female sex and treatment with DPP-4, insulin, statins, ezetimibe, calcium 
A) Univariate analysis of PFT, dds ratio of subject characteristics

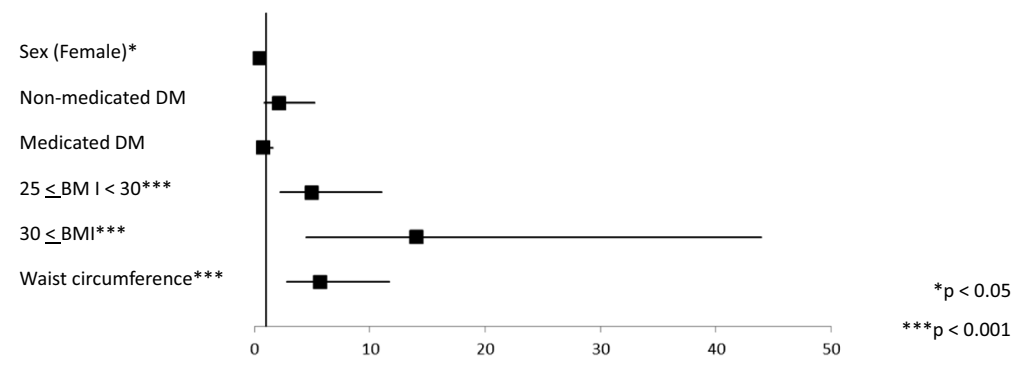

B) Univariate analysis of PFT, Odds ratio of therapeutic agents

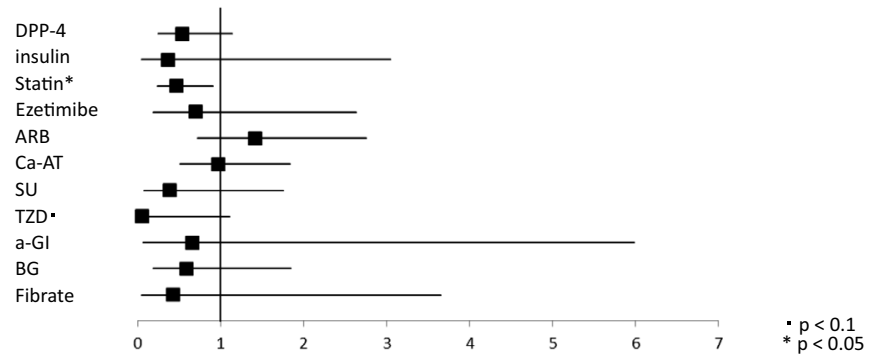

Figure 4. A: Univariate analysis: Odds ratios for the PFT based on the patient characteristics. Factors decreasing the risk of PFT included a female sex and medicated DM; the difference was significant for a female sex $(\mathbf{p}<0.05)$. Aggravating factors were established to be non-medicated DM, BMI and WC. Significant differences were found for a BMI and WC over the criteria $(\mathbf{p}<0.0001)$. B: Univariate analysis: Odds ratios for the PFT based on therapeutic agents. All medications for diabetes had an inhibitory effect on the PFT. Ca-ATs had no effect, while ARBs increased the risk of PFT. Significant differences were observed for statins $(\mathbf{p}<0.05)$ and TZD $(\mathbf{p}<0.1)$. ARB: angiotensin II receptor blocker, Ca-AT: calcium antagonist, DPP-4 : dipeptidyl-peptidase IV inhibitor, TZD: thiazolidinedione, BG: biguanide, a-GI: alpha-glucosidase inhibitor, SU: sulfonylurea

A) Univariate analysis of fatty liver disease, Odds ratio of subject characteristics

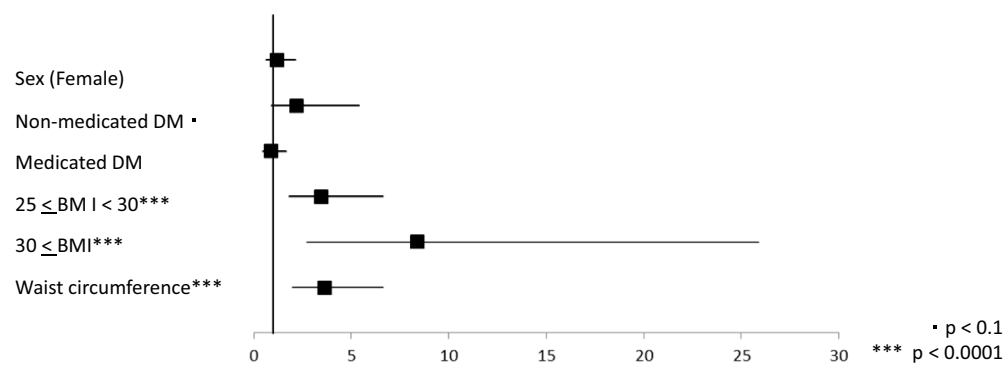

B) Univariate analysis of fatty liver disease, Odds ratio of therapeutic agents

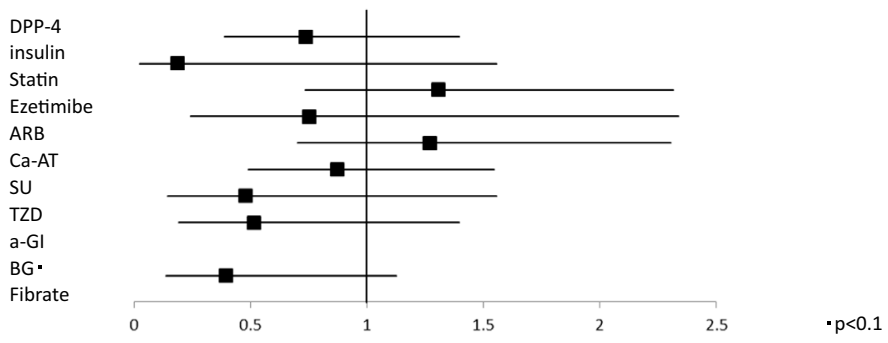

Figure 5. A: Univariate analysis: Odds ratios for the incidence of fatty liver disease based on the patient characteristics. The risk of fatty liver disease was significantly increased by the following factors: non-medicated DM $(p<0.1)$ and both a BMI and WC over the criteria $(p<0.0001)$. B: Univariate analysis: Odds ratios for the incidence of fatty liver disease based on the therapeutic agents. Statins and ARBs aggravated fatty liver disease, whereas DPP-4, insulin, ezetimibe, SUs, TZD and BGs each had an inhibitory effect. A significant difference was obtained for BGs (p<0.1). ARB: angiotensin II receptor blocker, Ca-AT: calcium antagonist, DPP-4: dipeptidyl-peptidase IV inhibitor, TZD: thiazolidinedione, BG: biguanide, a-GI: alpha-glucosidase inhibitor, SU: sulfonylurea 
Table 2. Multi-logistic Analysis (PFT)

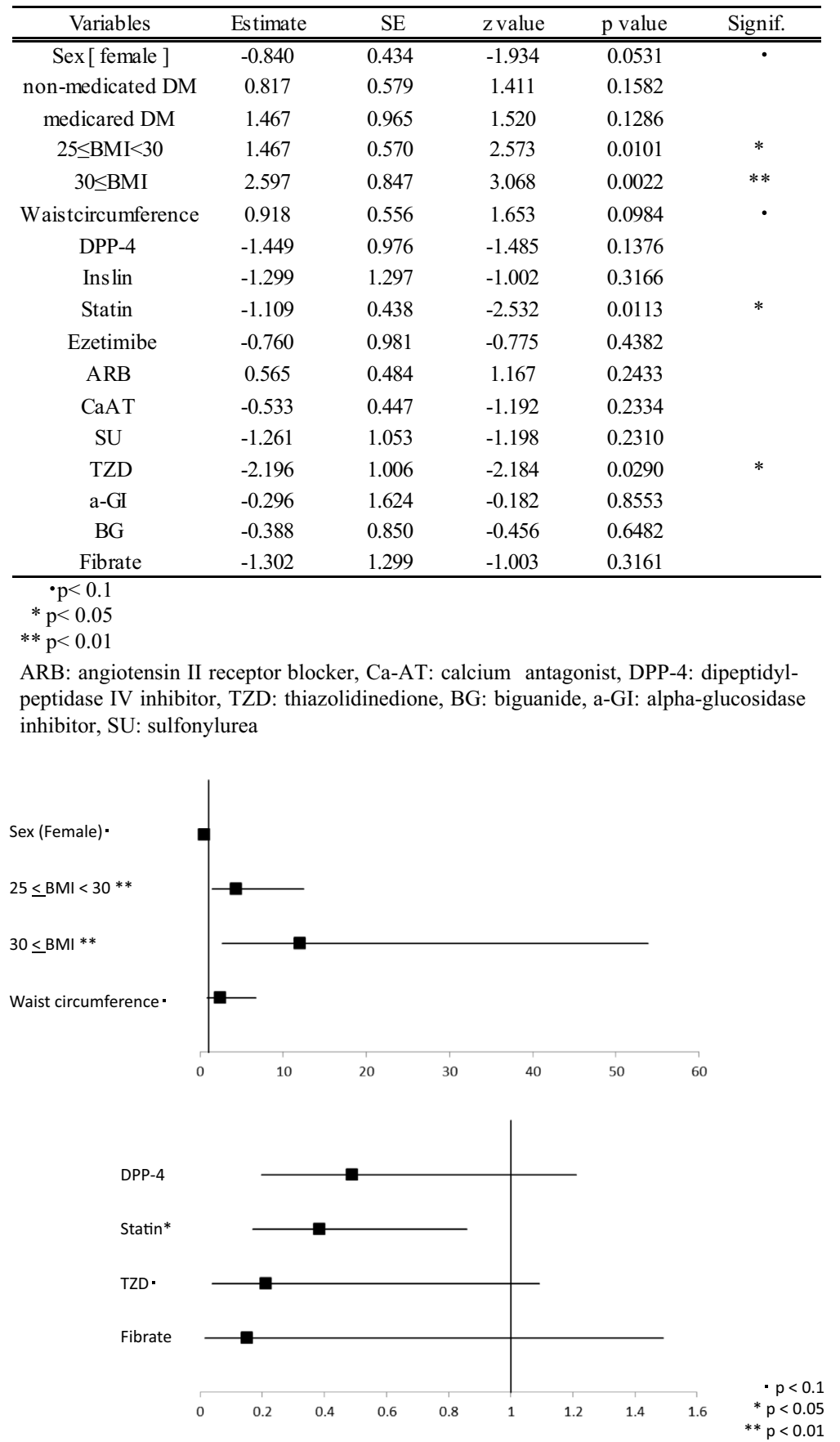

Figure 6. Stepwise method following a multiple logistic analysis (PFT). Aggravating factors for the PFT included a BMI and WC over the criteria. The following factors had an inhibitory effect on the PFT: a female sex and the use of DPP-4, statins, TZD or fibrates; significant differences were obtained for statins (estimate=-0.960, OR=0.383, $p=0.0199$ ) and TZD (estimate=-1.562, OR=0.21, p=0.0635). DPP-4: dipeptidyl-peptidase IV inhibitor, TZD: thiazolidinedione

antagonists, SUs, $\alpha$-GI inhibitors, BGs or bezafibrates.

The multivariate analysis revealed that the following factors significantly contributed to increased visceral fat accumulation: mild obesity (estimate $=1.463, \mathrm{OR}=4.319, \mathrm{p}=$ 0.0066 ), moderate or severe obesity (estimate $=2.483$, $\mathrm{OR}=$ 11.977, $\mathrm{p}=0.0012$ ) and waist circumference (estimate $=$ 0.882, $\mathrm{OR}=2.415, \mathrm{p}=0.0883$ ). The following factors had an inhibitory effect on visceral fat accumulation: a female sex (estimate $=-0.723, \mathrm{OR}=0.485, \mathrm{p}=0.0802$ ) and DPP-4 (estimate $=-0.716, \quad \mathrm{OR}=0.489, \mathrm{p}=0.1218$ ), statin (estimate $=-0.960, \quad \mathrm{OR}=0.383, \mathrm{p}=0.0199), \mathrm{TZD}$ (estimate $=-1.562$, $\mathrm{OR}=0.21, \mathrm{p}=0.0635$ ) or fibrate (estimate $=-1901, \mathrm{OR}=0.149$, $\mathrm{p}=0.1053$ ) use; significant differences were obtained for statins and TZD (Fig. 6).

The multiple logistic regression analysis of fatty liver disease revealed that an increased incidence of fatty liver dis- 
Table 3. Multi-logistic Analysis (Fatty Liver Disease)

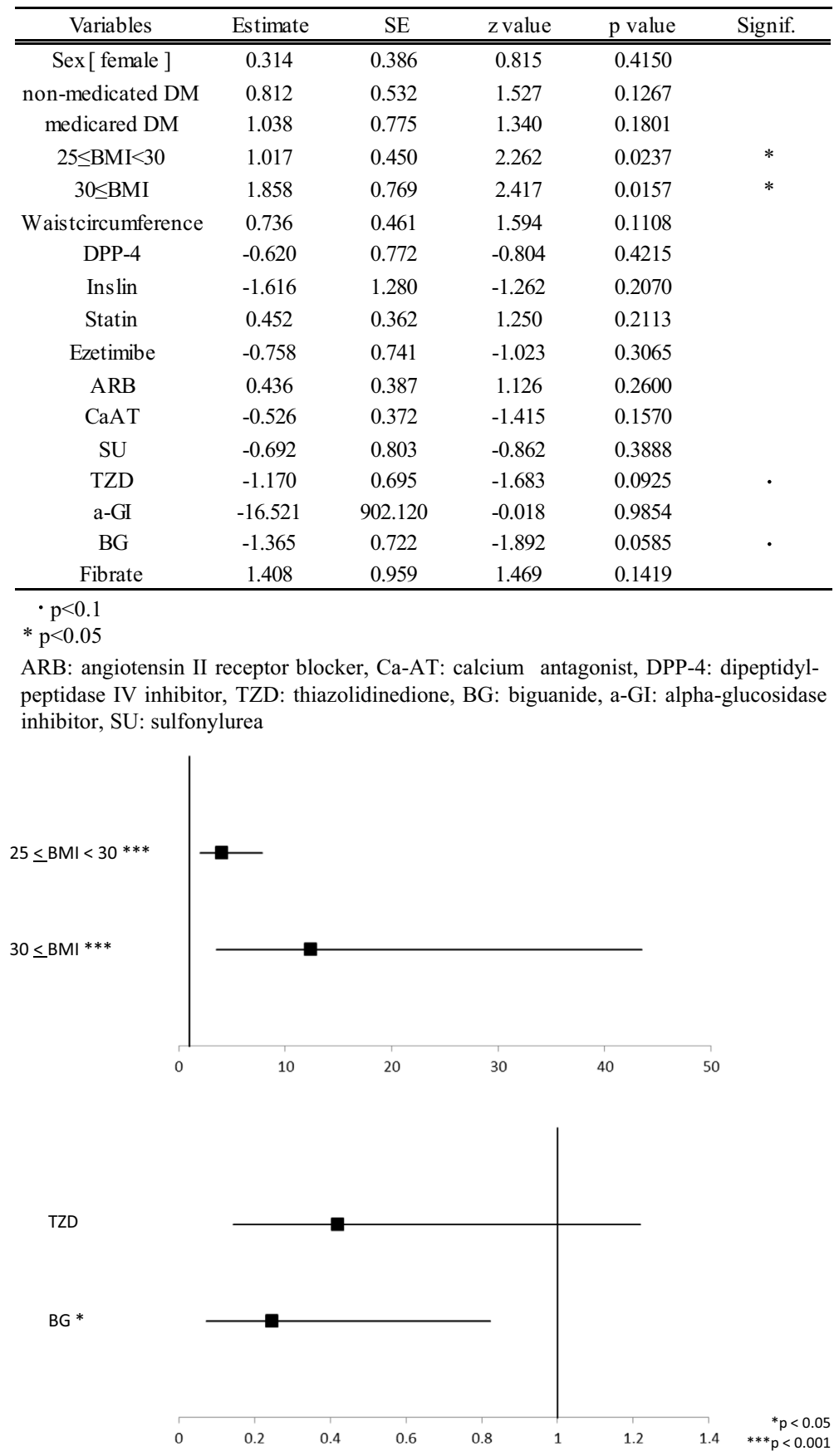

Figure 7. Stepwise method following a multiple logistic analysis (fatty liver disease). The multivariate analysis revealed that a BMI over the criteria was an aggravating factor, whereas the use of TZD or BGs was an inhibitory factor; a significant difference was observed for BGs (estimate=-1.405, OR=0.245, p=0.0227). TZD: thiazolidinedione, BG: biguanide

ease was correlated with the following factors: a female sex, diabetic medication use, obesity, a waist circumference over the cutoff point and statin, ARB or fibrate use. In contrast, the following factors had an inhibitory effect: a male sex and the use of DPP-4, insulin, ezetimibe, calcium antagonists, SUs, $\alpha$-GI inhibitors or BGs (Table 3). The multivariate analysis revealed that mild obesity (estimate $=1.392$, $\mathrm{OR}=4.024, \mathrm{p}<0.0001)$ and moderate or severe obesity (estimate $=2.516, \mathrm{OR}=12.375, \mathrm{p}<0.0001$ ) were aggravating fac- tors, whereas TZD (estimate $=-0.867, \mathrm{OR}=0.42, \mathrm{p}<0.145$ ) and BGs (estimate $=-1.405, \mathrm{OR}=0.245, \mathrm{p}<0.0227$ ) both had inhibitory effects; a significant difference was observed for BGs (Fig. 7).

\section{Discussion}

The Onitsuka Clinic, a peripheral medical institution and associated pharmacy, is located in the southern part of $\mathrm{Na}-$ 
gasaki City and serves a limited clinical population. In this cohort, it is notable that there is a large number of elderly patients as well as a preponderance of women. Adherence to treatment is excellent because these patients inevitably go regularly to the same clinic over many years due to the large proportion of senior patients.

In this study, the medicated DM group had the lowest LDL cholesterol levels, likely because these patients were taking statins to prevent the development of vascular complications; although no significant differences were found, a real trend was observed. In addition, many of the patients were taking medications with a low risk of hypoglycemia, allowing for good compliance; few patients were receiving $\alpha$-GI inhibitors or glinides that must be taken immediately before eating, and comparatively few patients were receiving SUs.

It is not possible to reach a conclusive diagnosis regarding a subject's risk of fatty liver based on BMI or a waist circumference of more than the standard value. However, both a high BMI and large waist circumference are major factors involved in the development of metabolic syndrome. Moreover, whether the visceral fat thickness or subcutaneous fat thickness is key cannot be accurately determined. However, instances of thick preperitoneal fat are often encountered during ultrasonography in diabetic patients with a normal BMI and waist circumference. Both preperitoneal and typical visceral fat are thought to originate from mesenteric fat. Therefore, an increased PFT corresponds to an increased amount of visceral fat (8-11).

In studies by Ma, Liu et al. and others, the amounts of subcutaneous fat, preperitoneal fat and mesenteric fat were measured using ultrasonographic examinations of the navel and the central part of the xiphoid process, and the thickness of preperitoneal and subcutaneous fat was found to contribute to insulin resistance and an enlarged waist circumference. The immediate measurement of mesenteric fat assumes that this parameter is a decisive element of metabolic syndrome based on the thickness of subcutaneous and preperitoneal fat in a similar way to the etiology of cardiovascular disease in which the presence of disease can be predicted based on measurements of the carotid intima-media thickness (IMT). It has also been reported that increased mesenteric fat is an independent determinant factor of fatty liver in patients with polycystic ovary syndrome (PCOS) $(12,13)$. Fatty liver is an emerging significant problem in PCOS patients in China, and the ultrasonic measurement of mesenteric fat has proven to be an independent determinant of fatty liver in such patients. Additionally, PCOS patients have been shown to have an increased risk of cardiovascular disease $(12,13)$. Certainly, it is believed that obtaining a direct measurement of mesenteric fat is useful for evaluating the presence of fatty liver and cardiovascular disease, and the PFT is one indicator of the amount of mesenteric fat. However, time and skill are required to obtain accurate measurements using this technique, and there is a possibility of differences in the skill of the operator resulting in variation in the measured values. In our study, the effect of the skill of the operator on the measurement of PFT was small; therefore, we believe that the ultrasonic diagnostic apparatus is suitable for widespread application, and, although it is inferior to the direct measurement of visceral fat, it is very useful for providing reasonably accurate results, and it also has the advantage that testing can be performed at the bedside during routine practice.

The prevalence of NAFLD/NASH will most likely increase in the future due to the continuing westernization of the diet and the increasing prevalence of chronic liver disease (1). Therefore, we believe that it is increasingly important to establish methods for preventing and treating NAFLD/NASH, particularly in the early stages of simple fatty liver disease, in order to prevent progression to NASH. Although weight loss achieved through proper diet and exercise is certainly the most effective approach, the administration of proper medications is essential.

In this study, the patients taking TZD had significantly lower PFT values, and, although no significant differences were found using a multivariate analysis, the prevalence of fatty liver disease tended to be low. In addition, the prevalence of fatty liver disease was significantly decreased in the patients taking BG. TZD inhibits fatty liver disease by acting as a ligand of peroxisome proliferator-activated receptor (PPAR- $\gamma$ ) and activating adiponectin and subsequently AMPK. BGs directly activate AMPK (5' adenosine monophosphate-activated protein kinase) to inhibit fatty acid synthesis (14-16). We believe that these drugs are highly promising medications for treating fatty liver disease in diabetic patients, particularly those without and not at high risk of developing obstructive sleep apnea syndrome or similar conditions in which resultant obesity is undesirable, congestive heart failure in which sodium retention is undesirable or bladder tumors $(17,18)$.

Although no significant differences were found, all other antidiabetic medications also inhibited an increase in the PFT and incidence of fatty liver disease. Therefore, lowering blood sugar levels alone may likely have an inhibitory effect, and whatever the precise mechanisms underlying insulin resistance, reducing glucotoxicity appears to be effective.

It is also possible that there was an interaction between the effects of the many medications used in this study. Several medications used to treat hyperlipidemia are known to have positive effects on metabolism and the development of NAFLD/NASH. We found that antidiabetic medication clearly contributes to the inhibition of fatty liver disease and visceral fat accumulation.

A female sex was found to be an inhibitory factor for PFT, but not fatty liver disease. This finding may have been due to the fact that many subjects were postmenopausal women. Dramatic changes in iron metabolism together with decreases in female hormones that would otherwise inhibit fatty liver disease allow the prevalence of the disease to increase in postmenopausal women relative to that observed in premenopausal women (19). 
The onset of NASH is influenced by a broad range of genetic factors, such as insulin resistance, as well as other factors, many of which still need to be studied. However, visceral fat, which secretes a variety of biologically active substances, is a known contributor to the onset of NAFLD/ NASH. Although obtaining accurate measurements with ultrasound is more difficult than with CT, in this study, ultrasound examinations were found to be simple, minimally invasive and useful in screening non-obese patients for visceral fat accumulation. Ultrasound can therefore play a role in evaluating the effectiveness of treatments for conditions other than diabetes that are influenced by visceral fat, such as cardiovascular disease, hypertension and dyslipidemia, as described by Liu et al. (12). This study also revealed that several medications are beneficial. In the present study, antidiabetic medications inhibited the development of fatty liver disease and visceral fat accumulation and ameliorated the effects of fatty liver disease. TZD and statins were effective in improving visceral fat accumulation. Furthermore, there is a possibility that DPP-4 and fibrates may also be useful, although further investigation is necessary. The usefulness of BGs for treating fatty liver disease was confirmed in this study, and our results suggested that TZD may also be useful.

We believe that measuring the PFT using ultrasonography can provide a more detailed understanding of the treatment status, making it a part of the treatment routine in diabetic patients, and, as such, this parameter could become a tool for controlling the development of NASH and reducing the incidence of the progression of hepatocellular carcinoma from simple fatty liver.

The authors state that they have no Conflict of Interest (COI).

\section{Acknowledgement}

The authors would like to thank the members of the Alumni Association of Kurume University School of Medicine in the Nagasaki district, who gave us the opportunity to carry out this study.

\section{References}

1. Shibata M, Kihara Y, Taguchi M, Tashiro M, Otsuki M. Nonalcoholic fatty liver disease is a risk factor for type 2 diabetes in middle-aged Japanese men. Diabetes Care 30: 2940-2944, 2007.

2. Day CP, James OFW. Steatohepatitis: a tale of two "hits"? Gastroenterology 114: 842-845, 1998.

3. Hashimoto E, Tokushige K. Prevalence, gender, ethnic variations, and prognosis of NASH. J Gastroenterol 46: 63-69, 2011.

4. Migoya EM, Stevens CH, Bergman AJ, et al. Effect of moderate hepatic insufficiency on the pharmacokinetics of sitagliptin. Can J Clin Pharmacol 16: e165-e170, 2009.

5. Maiztegui B, Borelli MI, Madrid VG, Del Zotto H, Raschia MA, Francini F. Sitagliptin prevents the development of metabolic and hormonal disturbances, increased beta-cell apotosis and liver steatosis induced by a fructose-rich diet in normal rats. Clin Sci 120: 73-80, 2011.

6. Shinkawa J, Fujii H, Ohnuma K, et al. Diet-induced adipose tissue inflammation and liver steatosis are prevented by DPP-4 inhibition in diabetic mice. Diabetes 60: 1246-1257, 2011.

7. The Examination Committee of Criteria for 'Obesity Disease' in Japan \& Japan Society for the Study of Obesity, 2002.

8. Uchibori E, Kadoh K, Nakamura H, et al. Preperitoneal fat thickness ( PFT ) of patients undergoing a medical examination assessed by ultrasonography method. Analysis of Biological Samples 34: 147-150, 2011 (in Japanese).

9. Suzuki R, Watanabe S, Hirai Y, et al. Abdominal wall index, estimated by ultrasonography, for assessment of the ratio of visceral fat to subcutaneous fat in the abdomen. Am J Med 95: 309-314, 1993.

10. Kim SK, Kim HJ, Hur KY, et al. Visceral fat thickness measured by ultrasonography can estimate not only visceral obesity but also risks of cardiovascular and metabolic disease. Am J Clin Nutr 79: 593-599, 2004.

11. Stolk RP, Meijer R, Mali W PTM, Grobbee DE, van der Graaf Y; SMART Study Group. Ultrasound measurements of intraabdominal fat estimate the metabolic syndrome better than do measurements of waist circumference. Am J Clin Nutr 77: 857-860, 2003.

12. Liu KH, Chan YL, Chan WB, Chan JCN, Chu CWW. Mesenteric fat thickness is an independent determinant of metabolic syndrome and identifies subjects with increased carotid intima-media thickness. Diabetes Care 29: 379-384, 2006.

13. Ma RCW, Liu KH, Lam PM, et al. Sonographic measurement of mesenteric fat predicts presence of fatty liver among subjects with polycytic ovary syndrome. J Clin Endocrinol Metab 96: 799-807, 2011.

14. Ratziu V, Caldwell S, Neuschwander-Tetri BA. Therapeutic trials in nonalcoholic steatohepatitis: Insulin sensitizers and related methodological issues. Hepatology 52: 2206-2215, 2010.

15. Marchesini G, Bianchi G, Tomassetti S, Zoli M, Melchionda N. Metformin in nonalcoholic steatohepatitis. Lancet 358: 893-894, 2001.

16. Belfort R, Harrison SA, Brown K, et al. A placebo-controlled trial of pioglitazone in subjects with nonalcoholic steatohepatitis. N Engl J Med 355: 2297-2307, 2006.

17. In: Risque de cancer de la vessie chez les personnes diabétiques traitées par pioglitazone en France: une étude de cohorte sur les données du SNIIRAM et du PMSI [Internet]. Scotte. afssaps.fr, Paris, 2011 [cited 2011 Sep.27]: 1-41 (in French) Available from: http://www.afssaps.fr/var/afssaps_site/storage/original/application/b 42a6bf9a1b63c3dbec7388d3914687b.pdf

18. Nesto RW, Bell D, Bonow RO, et al. Thiazolidinedione use, fluid retention, and congestive heart failure. Diabetes Care 27: 256-263, 2004.

19. Kim C, Nan B, Kong S, et al. Changes in iron measures over menopause and associations with insulin resistance. J Womens Health 21: 872-877, 2012.

(C) 2014 The Japanese Society of Internal Medicine http://www.naika.or.jp/imonline/index.html 